This item was submitted to Loughborough's Research Repository by the author.

Items in Figshare are protected by copyright, with all rights reserved, unless otherwise indicated.

\title{
Spectra of low-frequency ground vibrations generated by high-speed trains on layered ground
}

PLEASE CITE THE PUBLISHED VERSION

http://www.multi-science.co.uk/lowfreq.htm

\section{PUBLISHER}

(c) Multi-Science Publishing

VERSION

VoR (Version of Record)

\section{LICENCE}

CC BY-NC-ND 4.0

\section{REPOSITORY RECORD}

Krylov, Victor V.. 2012. "Spectra of Low-frequency Ground Vibrations Generated by High-speed Trains on Layered Ground”. figshare. https://hdl.handle.net/2134/10046. 
This item was submitted to Loughborough's Institutional Repository (https://dspace.lboro.ac.uk/) by the author and is made available under the following Creative Commons Licence conditions.

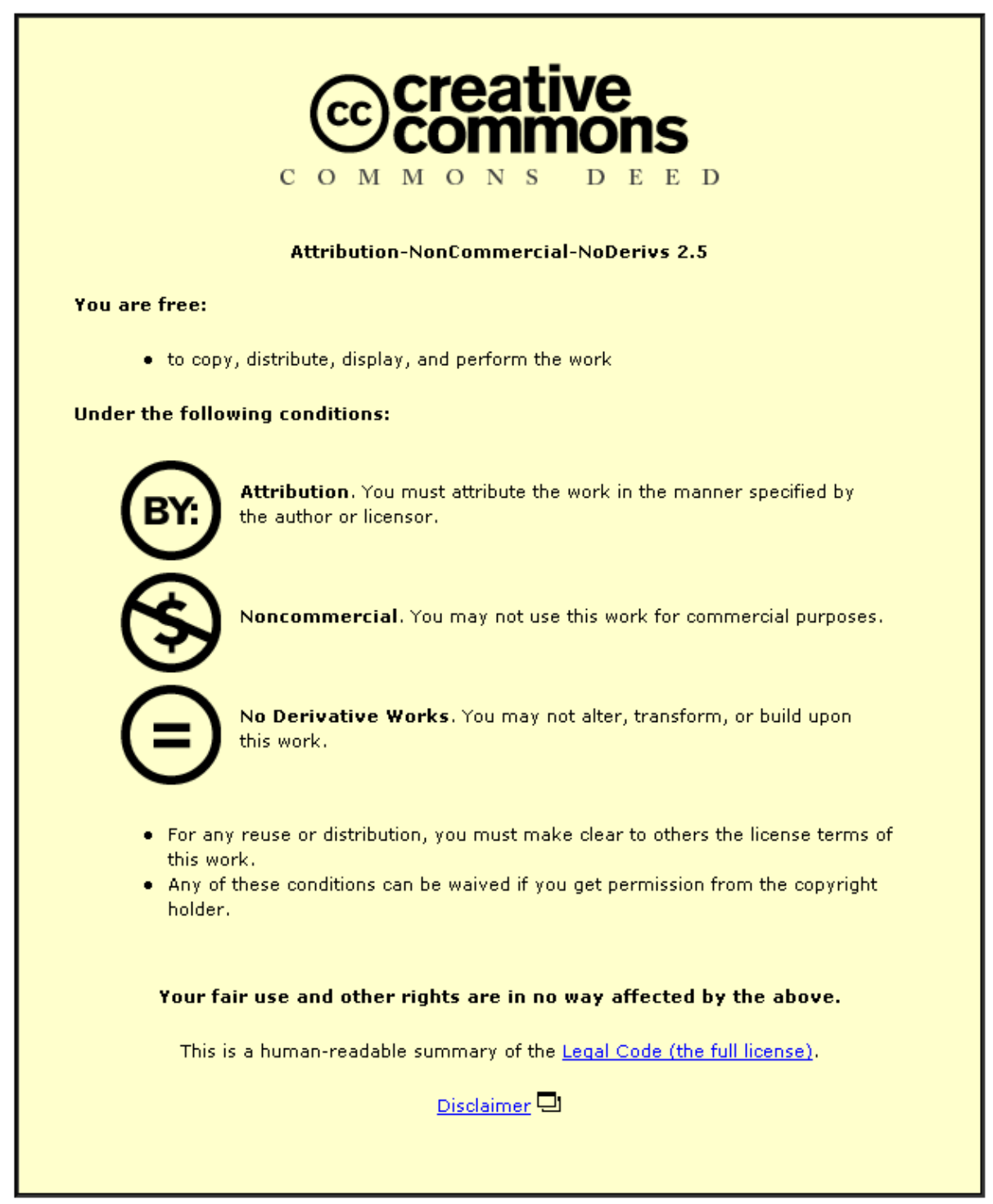

For the full text of this licence, please go to: http://creativecommons.org/licenses/by-nc-nd/2.5/ 
Spectra of Low-Frequency Ground Vibrations Generated by HighSpeed Trains on Layered Ground

by

V.V. Krylov

Reprinted from

\section{JOURNAL OF \\ LOW FREQUENCY NOISE, VIBRATION AND ACTIVE CONTROL}

VOLUME 16 No. 41997

MULTI-SCIENCE PUBLISHING CO. LTD.

107 High Street, Brentwood, Essex CM14 4RX, United Kingdom 


\title{
Spectra of Low-Frequency Ground Vibrations Generated by High-Speed Trains on Layered Ground
}

\author{
V.V. Krylov \\ Centre for Research into the Built Environment, The Nottingham Trent \\ University, Burton Street, Nottingham NG1 4BU, UK
}

Received 14 August 1997

\begin{abstract}
Increase in speeds of modern railway trains is usually accompanied by higher levels of generated ground vibrations. In the author's earlier paper [V.V. Krylov, Applied Acoustics, 44, 149-164 (1995)], it has been shown that especially large increase in vibration level may occur if train speeds $v$ exceed the velocity of Rayleigh surface waves in the ground $c_{R}$, i.e., $v>c_{R}$. Such a situation might arise, for example, with French TGV trains for which speeds over $515 \mathrm{~km} / \mathrm{h}$ have been achieved. The present paper investigates the effect of geological layered structure of the ground on ground vibrations generated by high-speed trains. It is shown that, since Rayleigh wave velocities in layered ground are dispersive and normally increase at lower frequencies associated with deeper penetration of surface wave energy into the ground, the transRayleigh condition $v>c_{R}$ may not hold at very low frequencies. This will cause a noticeable reduction in low-frequency components of generated ground vibration spectra. Theoretical results are illustrated by numerically calculated frequency spectra of ground vibrations generated by single axle loads travelling at different speeds and by TGV or Eurostar high-speed trains.
\end{abstract}

\section{INTRODUCTION}

According to a number of practical observations, an increase in speeds of modern trains is usually accompanied by increased levels of generated ground vibrations and associated structure-borne noise, including those at low and very low frequencies. We remind the reader that railway-generated ground vibrations are significant even for conventional passenger and heavy-freight trains travelling both above- and underground (Remington et al., 1987; Newland \& Hunt, 1991; Dawn, 1983; Volberg, 1983; Melke, 1988; Jones, 1994; Krylov \& Ferguson, 1994; Krylov, 1994). Recent theoretical investigations of ground vibrations from high-speed trains carried out by the present author (Krylov, 1994, 1995, 1996) contributed to understanding the reasons why an increase in train speeds is generally accompanied by higher levels of generated ground vibrations. In addition to this, it has been predicted that an especially large increase in vibration level (more than $70 \mathrm{~dB}$, as compared to conventional trains) should take place if train speeds $\mathrm{v}$ exceed the velocity of Rayleigh surface waves in the ground $c_{R}$ (such trains are called "trans-Rayleigh trains" in this paper). The condition $v>c_{R}$, which is similar to that of supersonic jets, can be met e.g., by French TGV trains or Eurostar trains travelling along tracks placed on relatively soft grounds.

In the present paper, we examine the effects of layered ground structure on 
ground vibrations generated by high-speed trains. The main aim of this investigation is to achieve a preliminary understanding of how layered structure may affect generated ground vibration spectra, in particular their low-frequency components.

In the following sections we describe the outline of the theory of generating ground vibrations by high speed trains and analyse the effects of layered ground structure on generated vibrations. Finally, we discuss the results of the numerical calculations of ground vibration frequency spectra generated by single axle loads travelling at different speeds and by complete TGV or Eurostar high-speed trains.

\section{OUTLINE OF THE THEORY}

\subsection{Main Generation Mechanism}

Among the mechanisms of generating ground vibrations by railway trains one can mention the wheel-axle pressure onto the track, the effects of joints in unwelded rails, the unevenness of wheels or rails, and the dynamically induced forces of carriage- and wheel-axle bending vibrations excited mainly by unevenness of wheels and rails. The most common generation mechanism is pressure of wheel axles onto the track. For very high quality tracks and wheels, this mechanism is probably a major contributor to the low-frequency vibration spectra (up to $50 \mathrm{~Hz}$ ). In this paper we take into account contribution of the wheel-axle pressure mechanism only, assuming that rails and wheels are ideally even and no carriage or wheel-axle vibrations are excited.

We assume that a train has $\mathrm{N}$ carriages and moves at speed $\mathrm{v}$ on a perfect welded track with sleeper periodicity d (Figure 1,a). The wheel-axle pressure generation mechanism being considered results in downward deflections of the track beneath each wheel axle (Figure 1,b). These deflections produce a wavelike motion along the track moving at speed $\mathrm{v}$ and resulting in distribution of the axle load over the sleepers involved in the deflection distance (Krylov \& Ferguson, 1994; Krylov, 1994, 1995). Thus, each sleeper acts as a vertical force applied to the ground during the time necessary for a deflection curve to pass through the sleeper. It is these forces that result in generating ground vibrations by passing trains in the framework of the wheel-axle pressure mechanism.

An important aspect of analysing the above discussed wheel-axle pressure mechanism is calculation of the track deflection curve as a function of the elastic properties of track and soil and of the magnitude of the axle load. Since the track deflection distance is greater than the distance between sleepers, one can treat a track (i.e. two parallel rails with periodically fastened sleepers) as an Euler - Bernoulli elastic beam of uniform mass $m_{0}$ lying on an elastic or viscoelastic half space $z>0$. For simplicity we assume that the uniform mass $m_{0}$ of the beam is formed entirely by the track (i.e., by rails and sleepers only), although in practice an adhered layer of ballast may form an additional mass.

Firstly we recall a quasi-static approach to the solution of this problem. The classical solution starts with the static beam equation that models the response of an elastic half space as a reaction of an elastic (Winkler) foundation which is proportional to the beam deflection magnitude w. If $\mathrm{E}$ and I are Young's modulus and the cross-sectional momentum of the beam, $\alpha$ is the proportionality coefficient of the elastic foundation, $\mathrm{x}$ is the distance along the beam and $\mathrm{T}$ is a vertical point force applied to the beam at $\mathrm{x}=0$, then the static equation has the form (Timoshenko, 1942)

$$
E I \partial^{4} w / \partial x^{4}+\alpha w=T \delta(x),
$$


where $\delta(\mathrm{x})$ is the Dirac's delta-function. The solution of (1) may be written as

$$
w^{s t}=\left(T / 8 E I \beta^{3}\right) \exp (-\beta|x|)[\cos (\beta x)+\sin (\beta|x|)],
$$

where $\beta=(\alpha / 4 \mathrm{EI})^{1 / 4}$. Index "st" in eqn (2) and in the expression to follow indicates values obtained in the quasi-static approximation. According to eqn (2), one can take $x_{0}{ }^{\text {st }}=\pi / \beta$ as the effective quasi-static track deflection distance.
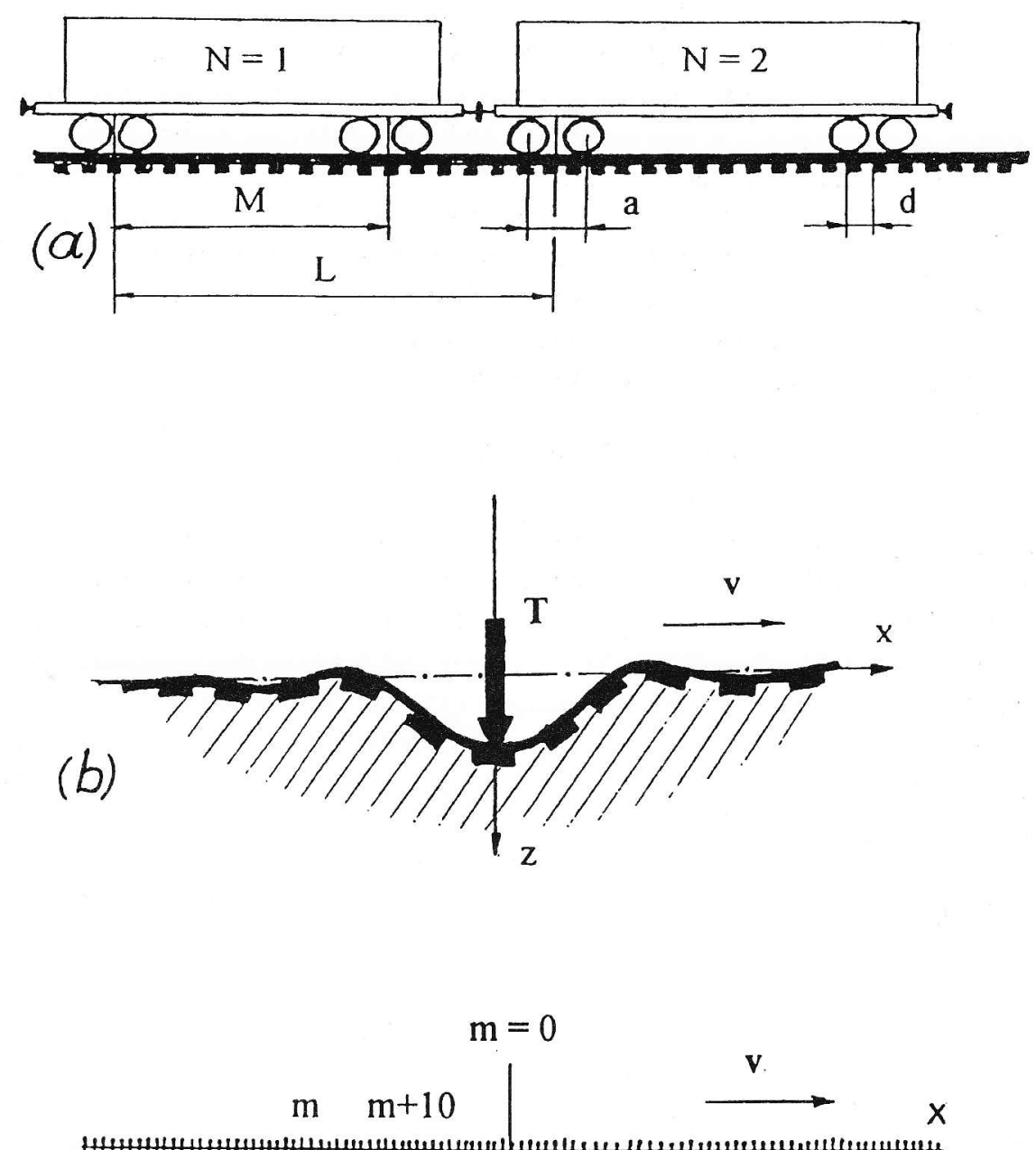

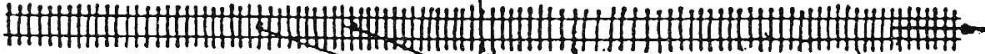

(C)

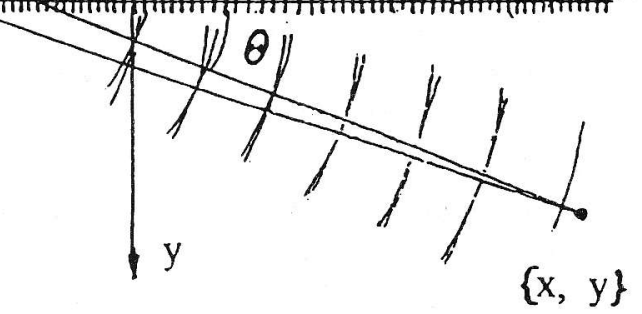

Figure 1. Geometrical parameters of track and train - (a); wheel-axle pressure mechanism - (b); superposition of ground vibrations generated by different sleepers at the point of observation $\{x, y\}-$ (c) 
The constant $\alpha$ in (1) and (2) depends on the stiffness of the ballast layer, of the ground and of the rubber pads inserted between rail and sleepers and under sleepers. In further consideration we assume that a well-compacted ballast layer is always present and is largely responsible for the proportionality coefficient $\alpha$ of the equivalent Winkler foundation corresponding to the combined system ballast/ground. The results of static track deflection tests show (Brockley, 1992) that, e.g., for British Rail tacks lying on a well-compacted ballasted roadbed, the coefficient $\alpha$ is determined mainly by the ballast layer. Typically $\alpha=52.6$ $\mathrm{MN} / \mathrm{m}^{2}$ and $\mathrm{EI}=4.85 \mathrm{MNm}^{2}$ (cited value of $\alpha$ also includes contribution of rubber pads characterised by distributed over a sleeper spacing coefficient of proportionality $\alpha_{\mathrm{rp}}=357 \mathrm{MN} / \mathrm{m}^{2}$ ). This results in $\beta=1.28 \mathrm{~m}^{-1}$. For a typical distance between sleepers, $d=0.7 \mathrm{~m}$, about seven sleepers are involved in the deflection distance $x_{0}{ }^{\text {st }}=\pi / \beta$ associated with each axle (see Figure 1,b).

After the track deflection curve having been determined, each sleeper may be considered as a vertical concentrated force applied to the ground surface $\mathrm{z}=0$, with time dependence determined by the passage of the deflection curve through the sleeper. For a sleeper resting on Winkler foundation and located at $\mathrm{x}=0$ the general expression for this force, which is valid for both quasi-static and dynamic regimes, may be written in the form

$$
P(t)=T\left[2 w(v t) / w_{\max }{ }^{s t}\right]\left(d / x_{0}{ }^{s t}\right)
$$

where $\mathrm{w}_{\max }{ }^{\mathrm{st}}$ is the maximal value of $\mathrm{w}(\mathrm{vt})$ in quasi-static approximation. To derive (3), one should take into account that $\mathrm{P}(\mathrm{t})$ is proportional to the track deflection $\mathrm{w}(\mathrm{vt})$ and to the sleeper width $\Delta \mathrm{d}: \mathrm{P}(\mathrm{t})=\alpha \mathrm{w}(\mathrm{vt}) \Delta \mathrm{d}$, where $\alpha$ is a constant of Winkler foundation. To exclude $\alpha$ and $\Delta \mathrm{d}$ from this expression, one should integrate the quasi-static equation (1) over $\mathrm{x}$. The integration, which takes into account that contact takes place only between sleepers and the ground, results in the formula $\alpha \mathrm{w}_{\max }{ }^{\text {st }} \Delta \mathrm{dN}_{\text {eff }}{ }^{\text {st }}=\mathrm{T}$ which, combined with the previous one, gives the following expression for $\mathrm{P}(\mathrm{t})$ :

$$
P(t)=T\left[w(v t) / w_{\max }{ }^{s t} N_{\text {eff }}{ }^{\text {st }}\right] \text {. }
$$

Here $\mathrm{N}_{\text {eff }}{ }^{\text {st }}$ is the effective number of sleepers equalising the applied quasi-static axle load $\mathrm{T}$ :

$$
\sum_{m=-N_{e f f}{ }^{s t} / 2}^{N_{e f f}{ }^{s t} / 2} \frac{T}{N_{e f f}^{s t}} \frac{w(m d)}{w_{\max }^{s t}}=T
$$

where $m$ denotes a number of a current sleeper. Numerical solution of eqn (4) shows that for $\beta$ within the range of interest (from $0.2 \mathrm{~m}^{-1}$ to $1.3 \mathrm{~m}^{-1}$ ) the value of $\mathrm{N}_{\text {eff }}^{\text {st }}$ may be approximated by a simple analytical formula $\mathrm{N}_{\text {eff }}{ }^{\mathrm{st}}=\pi / 2 \beta \mathrm{d}=$ $\mathrm{x}_{\mathrm{o}}$ st/2d. Using this formula in the expression for $\mathrm{P}(\mathrm{t})$ results in eqn (3). For highspeed passenger trains it may happen that train speeds $\mathrm{v}$ become of the same order as the minimal phase velocity $c_{\min }$ of dispersive bending waves propagating in the system track/ballast. In this case one can expect that dynamic effects may play a noticeable role in determining the track deflection curve. To calculate dynamic forces applied from sleepers to the ground one can continue to use the model of Winkler foundation, as is usually being done for quasi-static loads (see above). Strictly speaking, such a replacement is valid only for slowly moving loads compared to the velocities of elastic waves in the ground. Although some authors use it for calculating rail deflections for much higher load speeds (e.g., Fryba, 1973; Belzer, 1988), one should be aware that validity of the corresponding results for real ground is at best qualitative. 
dynamic equation of a beam on an elastic foundation (see, e.g., Belzer, 1988):

$$
E I \partial^{4} w / \partial x^{4}+m_{0} \partial^{2} w / \partial t^{2}+\alpha w=T \delta(x-v t)
$$

The solution of (5), which generalises the static solution (2), has the form (Belzer, 1988)

$$
w(x-v t)=\left(T / 8 E I \beta^{3} \delta\right) \exp (-\beta \delta \mid x-v t)[\cos (\beta \eta(x-v t)+(\delta / \eta) \sin (\beta \eta|x-v t|)]
$$

Here $\delta=\left(1-v^{2} / c^{2}{ }_{\min }\right)^{1 / 2}$ and $\left.\eta=1+v^{2} / c^{2}{ }_{\min }\right)^{1 / 2}$, the term $c_{\min }=\left(4 \alpha E I / m_{o}{ }^{2}\right)^{1 / 4}$ represents the minimal phase velocity of bending waves propagating in a system track/ground. For EI $=4.85 \mathrm{MNm}^{2}, \alpha=52.6 \mathrm{MN} / \mathrm{m}^{2}$ and $\mathrm{m}_{0}=300 \mathrm{~kg} / \mathrm{m}$ $\mathrm{c}_{\min }=326 \mathrm{~m} / \mathrm{s}(1174 \mathrm{~km} / \mathrm{h})$. Note that, for typical parameters of track and ballast, the above-mentioned value of $c_{\min }$ is essentially larger than even the highest train speed $(v=515 \mathrm{~km} / \mathrm{h})$. However, for soft marshy soils or for specially designed vibro-isolated tracks the value of $\mathrm{c}_{\min }$ may be much lower.

To calculate the forces applied from sleepers to the ground one should substitute eqn (6) into eqn (3) which is valid for both quasi-static and dynamic regimes. Since the factor $\delta=\left(1-\mathrm{v}^{2} / \mathrm{c}^{2}{ }_{\min }\right)^{1 / 2}$ is present in the denominator of the expression following from eqns (6) and (3), these forces increase as the train speed approaches the minimal track wave velocity (note that in our earlier paper (Krylov, 1996) the factor $\delta$ has been mistakenly written in the nominator that caused an opposite result).

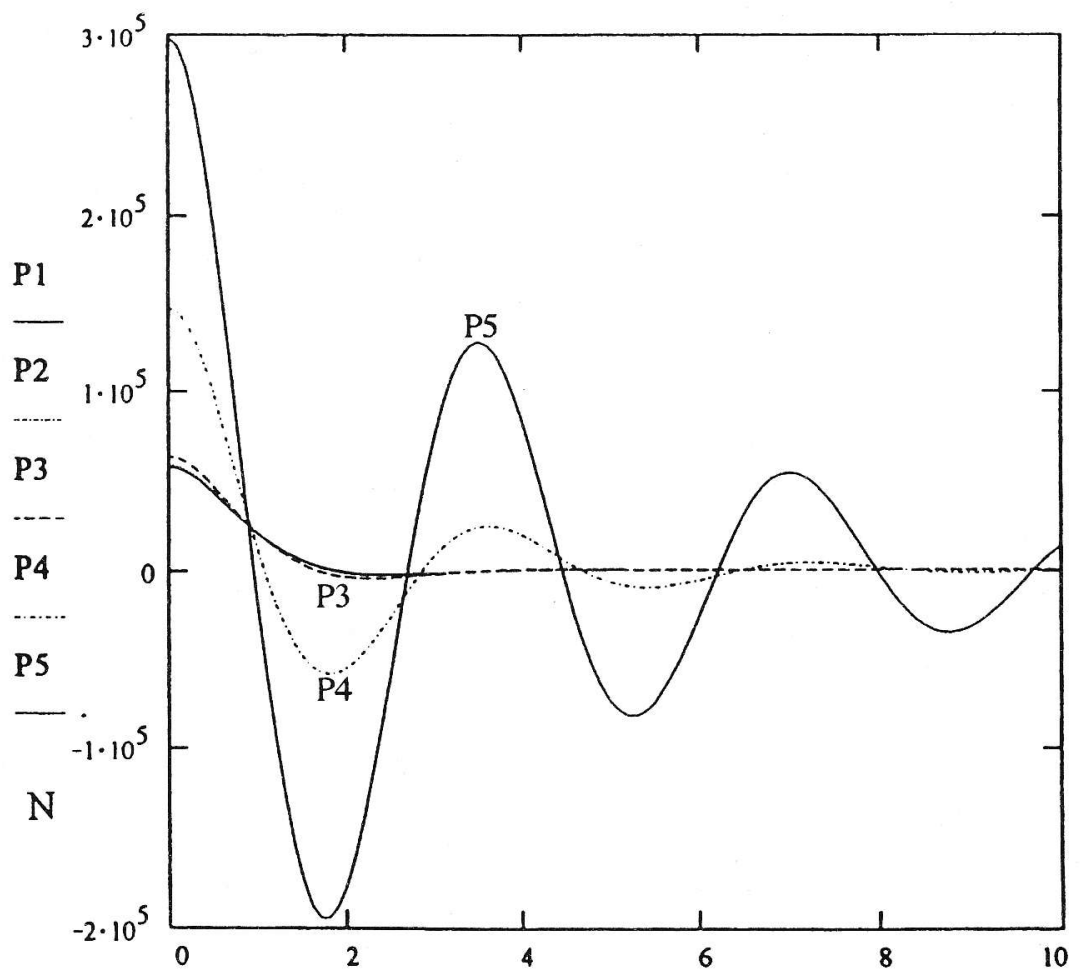

Figure 2. Vertical load forces applied from each sleeper to the ground as functions of vt for axle loads $\mathrm{T}=100 \mathrm{kN}$ moving along the track at speeds $0,69,138,300$ and $320 \mathrm{~m} / \mathrm{s}$ (curves P1, P2, P3, P4 and $P 5$ respectively); critical track wave velocity $c_{\min }$ is $326 \mathrm{~m} / \mathrm{s}$, distance between sleepers $d$ is $0.7 \mathrm{~m}$ 
The load forces $\mathbf{P}$ applied from each sleeper to the ground and calculated according to eqns (6) and (3) for $\mathrm{v}<\mathrm{c}_{\min }$ are shown in Figure 2 as functions of $\mathrm{vt}$ for the axle load $\mathrm{T}=100 \mathrm{kN}$ and for the values of train speed $\mathrm{v}$ equal to 0 , $69,138,300$ and $320 \mathrm{~m} / \mathrm{s}$ (curves P1-P5 respectively). One can see that the curves corresponding to the first three values of train speed (i.e., up to 500 $\mathrm{km} / \mathrm{h}$ ) are almost indistinguishable. Only for speeds approaching the minimal (critical) track wave velocity $c_{\min }=326 \mathrm{~m} / \mathrm{s}$ a significant increase occurs.

\subsection{GREEN'S FUNCTION FORMALISM}

The Green's function for the problem under consideration describes ground vibrations generated by individual sleepers which can be regarded as point sources in the low-frequency band. We recall that for homogeneous elastic half space the corresponding Green's function can be derived using the results from the well-known axisymmetric problem for the excitation of an elastic half space by a vertical point force applied to the surface (see, e.g., Ewing et al., 1957; Graff, 1975). The solution of this problem, which should satisfy the dynamic equations of elasticity for a homogeneous medium subject to the stress-free boundary conditions on the surface, gives the corresponding components of the dynamic Green's tensor (or, for simplicity, the Green's function) $G_{z i}$ for an elastic half space. For the problem under consideration, only Rayleigh surface wave contribution (the Rayleigh part of the Green's function) is considered, since Rayleigh waves transfer most of the vibration energy to remote locations. For these waves the spectral density of the vertical vibration velocity at the surface of homogeneous half space $(\mathrm{z}=0)$ may be written in the form (see below Krylov \& Ferguson, 1994)

$$
v_{z}(\rho, \omega)=P(\omega) G_{z z}(\rho, \omega)=P(\omega) D(\omega)\left(1 / \mathcal{V}_{\rho}\right) \exp \left(i k_{R} \rho-\gamma k_{R} \rho\right),
$$

where

$$
D(\omega)=(\pi / 2)^{1 / 2}(-i \omega) q k_{R}^{1 / 2} k_{1}^{2} \exp (-i 3 \pi / 4) / \mu F^{\prime}\left(k_{R}\right) .
$$

Here $\rho=\left[\left(x-x^{\prime}\right)^{2}+\left(y-y^{\prime}\right)^{2}\right]^{1 / 2}$ is the distance between the source (with current coordinates $x^{\prime}, y^{\prime}$ ) and the point of observation (with coordinates $x, y$ ), $\omega=2 \pi F$ is a circular frequency, $k_{R}=\omega / c_{R}$ is the wavenumber of a Rayleigh surface wave, $c_{R}$ is the Rayleigh wave velocity, $k_{1}=\omega / c_{1}$ and $k_{t}=\omega / c_{t}$ are the wavenumbers of longitudinal and shear bulk elastic waves, where $c_{t}=\left[(\lambda=2 \mu) / \rho_{0}\right]^{1 / 2}$ and $c_{t}=\left(\mu / \rho_{0}\right)^{1 / 2}$ are longitudinal and shear wave velocities, $\lambda$ and $\mu$ are the elastic Lame' constants, $\rho_{\mathrm{o}}$ is mass density of the ground, and $\mathrm{q}=\left(\mathrm{k}_{\mathrm{R}}{ }^{2}-\mathrm{k}_{1}^{2}\right)^{1 / 2}$. The factor $\mathrm{F}^{\prime}\left(\mathrm{k}_{\mathrm{R}}\right)$ is a derivative of the so-called Rayleigh determinant

$$
F(k)=\left(2 k^{2}-k_{l}^{2}\right)^{2}-4 k^{2}\left(k^{2}-k_{t}^{2}\right)^{1 / 2}\left(k^{2}-k_{l}^{2}\right)^{1 / 2}
$$

taken at $\mathrm{k}=\mathrm{k}_{\mathrm{R}}$, and $\mathrm{P}(\omega)=\left(\frac{1}{2 \pi}\right) \int_{-\infty}^{\infty} \mathrm{P}(\mathrm{t}) \exp (\mathrm{i} \omega \mathrm{t}) \mathrm{dt}$ is a Fourier transform of
$\mathrm{P}(\mathrm{t})$. Taking the Fourier transforms of (6) and (3) at $\mathrm{x}=0$, one can easily obtain the corresponding formula for $\mathrm{P}(\omega)$ : 


$$
\begin{aligned}
P(\omega)= & \left(T \beta d / \pi^{2} \delta\right)\left\{[\beta \delta v+(\beta \eta v+\omega)] /\left[(\beta \delta v)^{2}+(\beta \eta v+\omega)^{2}\right]+\right. \\
& {\left.[\beta \delta v+(\beta \eta v-\omega)] /\left[(\beta \delta v)^{2}+(\beta \eta v-\omega)^{2}\right]\right\} . }
\end{aligned}
$$

To describe the spectrum for successive passage of two axle loads separated by the distance a (the case of a bogie), $\mathrm{P}_{\mathrm{b}}(\omega)$, one should use the following relationship between $\mathrm{P}_{\mathrm{b}}(\omega)$ and $\mathrm{P}(\omega)$ (Krylov \& Ferguson, 1994):

$$
P_{b}(\omega)=2 P(\omega) \cos (\omega a / 2 v) .
$$

In writing (7) we have accounted for attenuation in soil by replacing $1 / c_{R}$ in the exponential of the Green's function by the complex value $1 / c_{R}+i \gamma / c_{R}$, where $\gamma=0.001-0.1$ is a constant describing the "strength" of dissipation of Rayleigh waves in soil.

Eqn (7) implies a linear frequency dependence of soil attenuation, in agreement with the experimental data (White, 1965; Gutovski \& Dym, 1976).

Now we consider the influence of layered geological structure of the ground on generating ground vibrations. To do so in a rigorous way, we had to use the Green's function for a layered elastic half space, instead of that for a homogeneous half space. As a rule, such a function, that would contain information about the total complex elastic field generated in a layered half space considered (including different modes of surface waves and modes radiating energy into the bulk (leaky waves)), cannot be obtained analytically. However, for description of railway-generated ground vibrations, the problem can be simplified by considering an approximate solution which takes into account the effects of layered structure on the amplitude and phase velocity of only the lowest order surface mode which goes over to a Rayleigh wave at higher frequencies (the propagating modes of higher order and leaky modes are generated less effectively by surface forces associated with sleepers).

We recall that in layered media surface waves become dispersive, i.e., their phase velocities $c_{R}$ depend of frequency: $c_{R}=c_{R}(\omega)$. For shear modulus of the ground $\mu$ normally having larger values at larger depths, there may be several surface modes characterised by different phase velocities and cut-off frequencies. As a rule, these velocities increase at lower frequencies associated with deeper penetration of surface wave energy into the ground (see, e.g., Biryukov et al., 1995; Jones \& Petyt, 1993). For simplicity, we will assume in further consideration that the Poisson ratio $\sigma$ of the layered ground and the mass density $\rho_{\mathrm{o}}$ are constant.

Taking the above mentioned into account and starting from the Green's function for a homogeneous half space $G_{z z}(\rho, \omega)$ (see eqns (7), (8)), we will construct its modification $\mathrm{G}_{z z}^{\mathrm{L}}(\rho, \omega)$, describing approximately the effects of layered medium on generation and propagation of a lowest order surface Rayleigh mode:

$$
\begin{aligned}
& v_{z}(\rho, \omega)=P(\omega) G_{z z}^{L}(\rho, \omega)=P(\omega) D^{L}(\omega)\left(1 / \gamma_{\rho}\right) \exp \left(i k_{R}{ }^{L} \rho-\gamma k_{R}{ }^{l} \rho\right), \\
& D^{L}(\omega)=(\pi / 2)^{1 / 2}(-i \omega) q^{L}\left(k_{R}^{L}\right)^{1 / 2}\left(k_{t}^{L}\right)^{2} \exp (-i 3 \pi / 4) / \mu^{L}(\omega) F_{L}^{\prime}\left(k_{R}{ }^{l}\right)
\end{aligned}
$$

Here $k_{R}{ }^{L}=\omega / c_{R}(\omega)$ is the wavenumber of a lowest order Rayleigh mode propagating with frequency-dependent velocity $c_{R}(\omega)$; terms $k_{1}^{L}=\omega / c_{1}^{L}(\omega)$ and $k_{t}^{L}=\omega / c_{t}^{L}(\omega)$ are "effective" wavenumbers of longitudinal and shear bulk 
elastic waves at given frequency $\omega$. These wavenumbers are inversely proportional to the averaged over the "effective" depth of Rayleigh wave penetration into the ground (close to Rayleigh wavelength) longitudinal $c_{1}{ }^{L}(\omega)$ and shear $c_{t}^{L}(\omega)$ wave velocities. In the model under consideration, these velocities and the corresponding "effective" shear modulus $\mu^{\mathrm{L}}(\omega)$ are expressed in terms of frequency-dependent Rayleigh wave velocity $c_{R}(\omega)$ using the well known relations:

$$
\begin{gathered}
c_{R}(\omega) / c_{t}{ }^{L}(\omega)=(0.87+1.12 \sigma) /(1+\sigma) \\
c_{l}{ }^{L}(\omega) / c_{l}{ }^{L}(\omega)=[(1-2 \sigma) / 2(1-\sigma)]^{1 / 2} \\
\mu^{L}(\omega)=\rho_{0}\left[c_{l}^{L}(\omega)\right]^{2} .
\end{gathered}
$$

The term $\mathrm{q}^{\mathrm{L}}$ is defined as $\mathrm{q}^{\mathrm{L}}=\left[\left(\mathrm{k}_{\mathrm{R}}{ }^{\mathrm{L}}\right)^{2}-\left(\mathrm{k}_{1}^{\mathrm{L}}\right)^{2}\right]^{1 / 2}$, and the factor $\mathrm{F}_{\mathrm{L}}{ }^{\prime}\left(\mathrm{k}_{\mathrm{R}}{ }^{\mathrm{L}}\right)$ is determined according to the following relationship (Biryukov et al., 1995):

$$
F_{L}^{\prime}\left(k_{R}^{L}\right)=N(\sigma)\left(k_{R}^{L}\right)^{3}
$$

where $\mathrm{N}(\sigma)$ is a dimensionless function of the Poisson ratio $\sigma$ (e.g., for $\sigma=$ 0.25 , the function $\mathrm{N}(\sigma)$ takes the value -2.3$)$.

The particular dependence of Rayleigh wave velocity on frequency, $c_{R}(\omega)$, is determined by the particular profile of layered ground. Determination of this velocity is a complex boundary-value problem which, generally speaking, requires numerical calculation. In our approach we will consider published values of the functions $c_{R}(\omega)$ using, where possible, their simple analytical approximations.

One can expect that the most significant effect of layered structure on generating ground vibrations by high-speed trains will be due to the wave phase variations caused by frequency-dependent Rayleigh wave velocities. In particular, the increase in Rayleigh wave velocities at low frequencies might violate the trans-Rayleigh condition responsible for generation of very intensive ground vibrations $\mathrm{v}>\mathrm{c}_{\mathrm{R}}$, thus causing a reduction in the lowfrequency components of generated ground vibration spectra.

To calculate ground vibrations generated by a train one needs superposition of waves generated by each sleeper activated by wheel axles of all carriages, with the time and space differences between sources (sleepers) being taken into account (see Figure 1,c).

Using the Green's function this may be written in the form (Krylov \& Ferguson, 1994)

$$
v_{z}(x, y, \omega)=\int_{-\infty-\infty}^{\infty} \int_{-\infty}^{\infty} P\left(x^{\prime}, y^{\prime}, \omega\right) G_{z z}^{L}(\rho, \omega) d x^{\prime} d y^{\prime},
$$

where $\mathrm{P}\left(\mathrm{x}^{\prime}, \mathrm{y}^{\prime}, \omega,\right)$ describes the space distribution of all load forces acting along the track in the frequency domain. This distribution can be found by taking a Fourier transform of the time and space dependent load forces $\mathrm{P}\left(\mathrm{t}, \mathrm{x}^{\prime}\right.$, $\left.\mathrm{y}^{\prime}=0\right)$ applied from the track to the ground. Note that the function $\mathrm{P}\left(\mathrm{t}, \mathrm{x}^{\prime}, \mathrm{y}^{\prime}=\right.$ 0 ) does not depend on layered structure of the ground and remains the same as for a homogeneous half space. 
at speed $\mathrm{v}$ along the track, this function has the form:

$$
P\left(t, x^{\prime}, y^{\prime}=0\right)=\sum_{m=-\infty}^{\infty} P\left(t-x^{\prime} / v\right) \delta\left(x^{\prime}-m d\right) \delta\left(y^{\prime}\right)
$$

where delta-function $\delta\left(\mathrm{x}^{\prime}-\mathrm{md}\right)$ takes the periodic distribution of sleepers into account. The Fourier transform of (19) is being written as follows

$$
P\left(x^{\prime}, y^{\prime}, \omega\right)=P(\omega) \exp \left(i \omega x^{\prime} / v\right) \sum_{n=-\infty}^{\infty} \delta\left(x^{\prime}-m d\right) \delta\left(y^{\prime}\right)
$$

Substitution of eqn (20) into (18) and taking eqns (3) and (6) into account results in the following formula for the vertical vibration velocity of Rayleigh waves generated at $\mathrm{z}=0, \mathrm{x}=0, \mathrm{y}=\mathrm{y}_{0}$ by a single axle load moving along the track at speed $v$ :

$$
v_{z}\left(x=0, y=y_{0}, \omega\right)=P(\omega) D^{L}(\omega) \sum_{m=-\infty}^{\infty} \exp \left[i(\omega / v) m d+(i-\gamma)\left(\omega / c_{R}(\omega)\right) \rho_{m}\right] / \sqrt{\rho_{m}},
$$

where $\rho_{\mathrm{m}}=\left[\mathrm{y}_{\mathrm{o}}{ }^{2}+(\mathrm{md})^{2}\right]^{1 / 2}$. Formula (21) shows that a single axle load moving at conventional speeds $\left(\mathrm{v}<\mathrm{c}_{\mathrm{R}}(\omega)\right)$ generates a quasi-discrete spectrum with frequency peaks close to $f_{p} s$, where $f_{p}=v / d$ is the so-called main passage frequency, and $s=1,2,3 \ldots$ Deviation from perfect discreteness results from the $\mathrm{i}(\omega / \mathrm{cR}(\omega)) \rho_{\mathrm{m}}$ term in eqn (21) which takes into account phase differences of waves propagated from each sleeper to the point of observation.

To take account of all axles and carriages one should use a more complicated load function:

$$
P\left(t, x^{\prime}, y^{\prime}=0\right)=\sum_{m=-\infty}^{\infty} \sum_{n=0}^{N-1} A_{n}\left[P\left(t-\left(x^{\prime}+n L\right) / v\right)+P\left(t-\left(x^{\prime}+M+n L\right) / v\right)\right] \delta\left(x^{\prime}-m d\right) \delta\left(y^{\prime}\right),
$$

where $\mathrm{N}$ is the number of carriages, $\mathrm{M}$ is the distance between the centres of bogies in each carriage and $\mathrm{L}$ is the total carriage length. Dimensionless quantity $A_{n}$ is an amplitude weight-factor to account for different carriage masses. For simplicity we assume all carriage masses to be equal $\left(A_{n}=1\right)$.

Taking the Fourier transform of (22), substituting it into (18) and making simple transformations, we obtain the following expression for the frequency spectra of vertical vibrations at $z=0, x=0$ and $y=y_{0}$ generated by a moving train:

$$
\begin{gathered}
v_{z}\left(x=0, y=y_{0}, \omega\right)=P(\omega) D^{L}(\omega) \sum_{m=-\infty}^{\infty} \sum_{n=0}^{N-1}\left[\exp \left(-\gamma \omega \rho_{m} / c_{R}(\omega)\right) / \sqrt{ } \rho_{m} l[1+\exp (i M \omega / v)]\right. \\
\exp \left(i(\omega / v)(m d+n L)+i\left(\omega / c_{R}(\omega)\right) \rho_{m}\right)
\end{gathered}
$$

The summation over $\mathrm{m}$ in (21) and (23) considers an infinite number of sleepers. However, the contribution of remote sleepers is small because of soil attenuation and cylindrical spreading of Rayleigh waves, and a few hundred sleepers are adequate for practical calculations.

The general expressions (21) and (23) derived above are applicable to trains moving at arbitrary speeds. However, for the specific case of "trans-Rayleigh trains", i.e., trains travelling at speeds higher than Rayleigh wave velocity in the ground, a separate analytical treatment can be done to elucidate the special 
The general expressions (21) and (23) derived above are applicable to trains moving at arbitrary speeds. However, for the specific case of "trans-Rayleigh trains", i.e., trains travelling at speeds higher than Rayleigh wave velocity in the ground, a separate analytical treatment can be done to elucidate the special features of the problem (Krylov, 1995). Not repeating the details of this treatment, which are the same as in the case of homogeneous ground, we conclude that maximum radiation of ground vibrations takes place if the train speed $\mathrm{v}$ and Rayleigh wave velocity $\mathrm{c}_{\mathrm{R}}(\omega)$ satisfy the following relation

$$
\cos \Theta=c_{R}(\omega) / v,
$$

where $\Theta$ is the observation angle. Since the observation angle $\Theta$ must be real $(\cos \Theta \leq 1)$, the train speed $v$ should be larger than Rayleigh wave velocity $c_{R}(\omega)$. In this case ground vibrations are generated as quasi-plane Rayleigh surface waves symmetrically propagating at angles $\Theta$ with respect to the track, and with amplitudes much larger than for "sub-Rayleigh trains".

\section{NUMERICAL CALCULATIONS AND DISCUSSION}

Calculations of ground vibrations generated by high-speed trains have been carried out according to eqns (21) or (23) for different values of train speed, different parameters characterising Rayleigh wave dispersion in layered ground, and for different geometrical and physical parameters of both track and train. Summation over $m$ in eqns (21) and (23) was carried out from $m=-150$ to $m=150$, the corresponding length of track being greater than the total train length NL and the attenuation distance of Rayleigh waves at the frequency band considered. The frequency-dependent Rayleigh wave velocity for layered media was approximated by the function

$$
c_{R}(\omega)=\left(c_{1}-c_{2}\right) \exp (-s \omega / 2 \pi)+c_{2}
$$

where $c_{1}$ and $c_{2}$ are values of $c(\omega)$ for $\omega=0$ and $\omega=\propto$ respectively, parameter $s$ describes the "strengths" of dispersion (it depends on the characteristic layer thickness and on the difference between elastic moduli in the depth and on the surface of the ground). The Poisson's ratio of soil was set at 0.25 , and the mass density $\rho_{\mathrm{o}}$ was $2000 \mathrm{~kg} / \mathrm{m}^{3}$.

Figure 3 shows surface graphs of the ground vibration spectra (in linear units, relative to the reference level of $10^{-9} \mathrm{~m} / \mathrm{s}$ ) generated by a single axle load $\mathrm{T}=100 \mathrm{kN}$ moving at speeds ranging from $10 \mathrm{~m} / \mathrm{s}$ to $320 \mathrm{~m} / \mathrm{s}$ for homogeneous (a) and layered (b) ground. the results are given for the frequency band $0-50$ $\mathrm{Hz}$ and for the value of ground attenuation $\gamma=0.05$. Units of calculation were $\Delta \mathrm{v}=10 \mathrm{~m} / \mathrm{s}$ and $\Delta \mathrm{F}=1 \mathrm{~Hz}$. Values of the parameter $\mathrm{s}$ in eqn (25) corresponding to homogeneous and layered ground were 10 and 0.1 respectively. Other parameters were: $\beta=1.28 \mathrm{~m}^{-1}, \mathrm{y}_{0}=30 \mathrm{~m}$.

One can see that with the increase of train speed the ground vibration level generally grows. For relatively low train speeds, the peaks corresponding to the train passage frequencies are almost invisible because of the huge increase of vibration level in the trans Rayleigh range $\left(v \geq c_{R}(\omega)\right)$. This increase goes even further for train speeds approaching the minimal track wave velocity $\left(\mathrm{c}_{\min }=\right.$ $326 \mathrm{~m} / \mathrm{s}$ in this example). Comparison of Figures 3,a and 3,b shows that effect of layered structure results in decrease of generated vibrations at low frequencies.

Figure 4 illustrates the ground vibration spectra (in $\mathrm{dB}$, relative to the 
reference level of $10^{-9} \mathrm{~m} / \mathrm{s}$ ) generated by complete French TGV trains or Eurostar trains travelling on homogeneous ground for both sub-Rayleigh and trans-Rayleigh train speeds (respectively $\mathrm{v}=50 \mathrm{~km} / \mathrm{h}$ - curve Vz1 and $\mathrm{v}=500$ $\mathrm{km} / \mathrm{h}$-curve Vz2), and for layered ground at the same train speeds (curves Vz3 and $\mathrm{Vz} 4$ respectively). Train consists of $\mathrm{N}=5$ equal carriages with the parameters $L=18.9 \mathrm{~m}$ and $M=15.9 \mathrm{~m}$ (see Figure 1,a). Since the bogies of TGV and Eurostar trains have a wheel spacing of $3 \mathrm{~m}$ and are placed between carriage ends, i.e., they are shared between two neighbouring carriages, to use the eqn (23) one should consider each carriage as having one-axle bogies $(\mathrm{a}=$ 0 ) separated by the distance $M=15.9 \mathrm{~m}$. Other parameters of track and ground used in calculations are the same as in Figure 3.
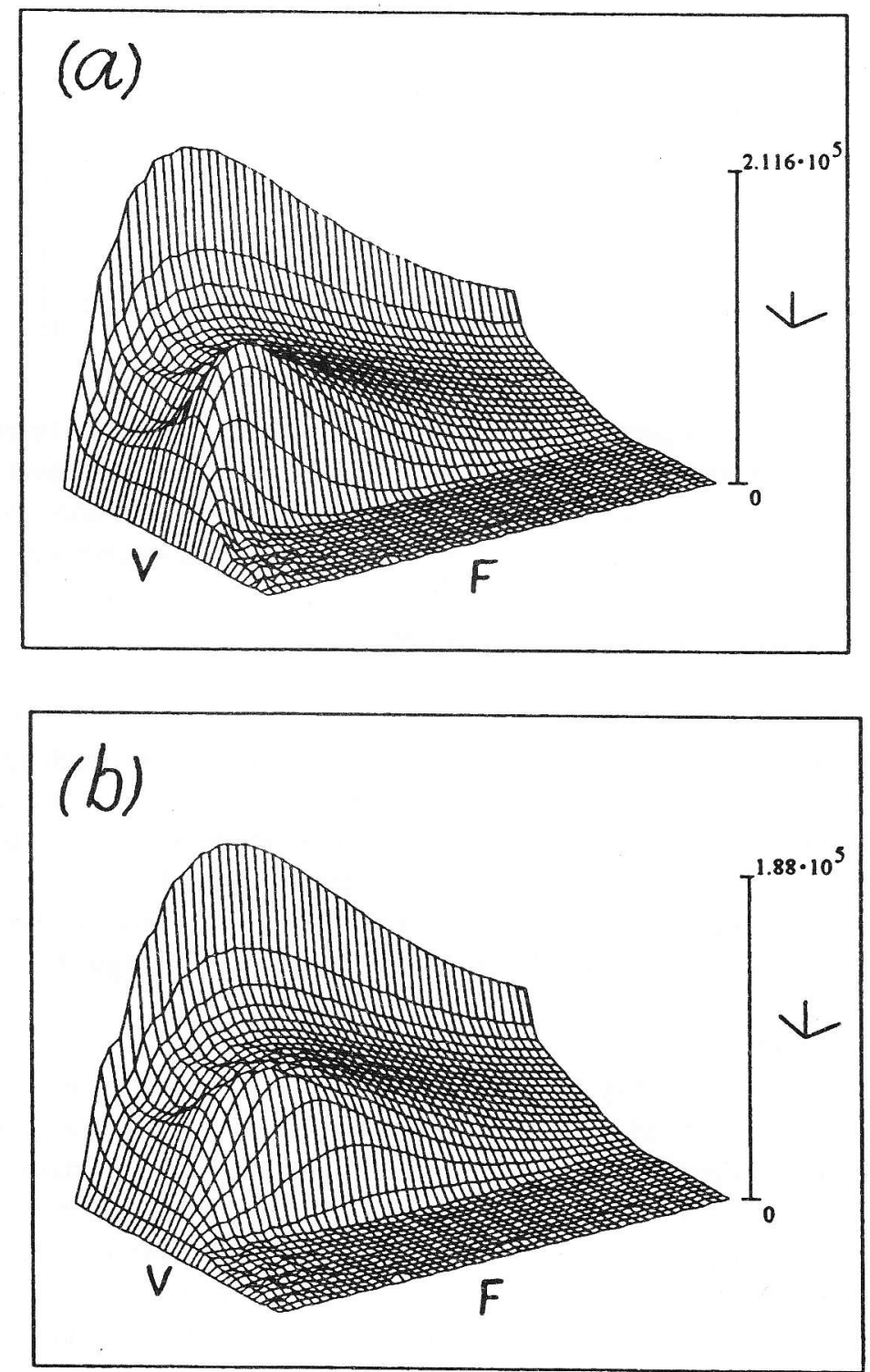

Figure 3. Spectra of ground vibration velocity (in linear units, relative to the reference level of $10^{-9} \mathrm{~m} / \mathrm{s}$ ) for a single axle load moving along the track at speeds from $10 \mathrm{~m} / \mathrm{s}$ to $320 \mathrm{~m} / \mathrm{s}$ on the surface of a homogeneous (a) and layered (b) half-space. The results are shown in the form of surface graphs for the frequency band $0-50$ Hz. Mesh: $\mathrm{Dv}=10 \mathrm{~m} / \mathrm{s}$ and $\mathrm{DF}=1 \mathrm{~Hz}$ 


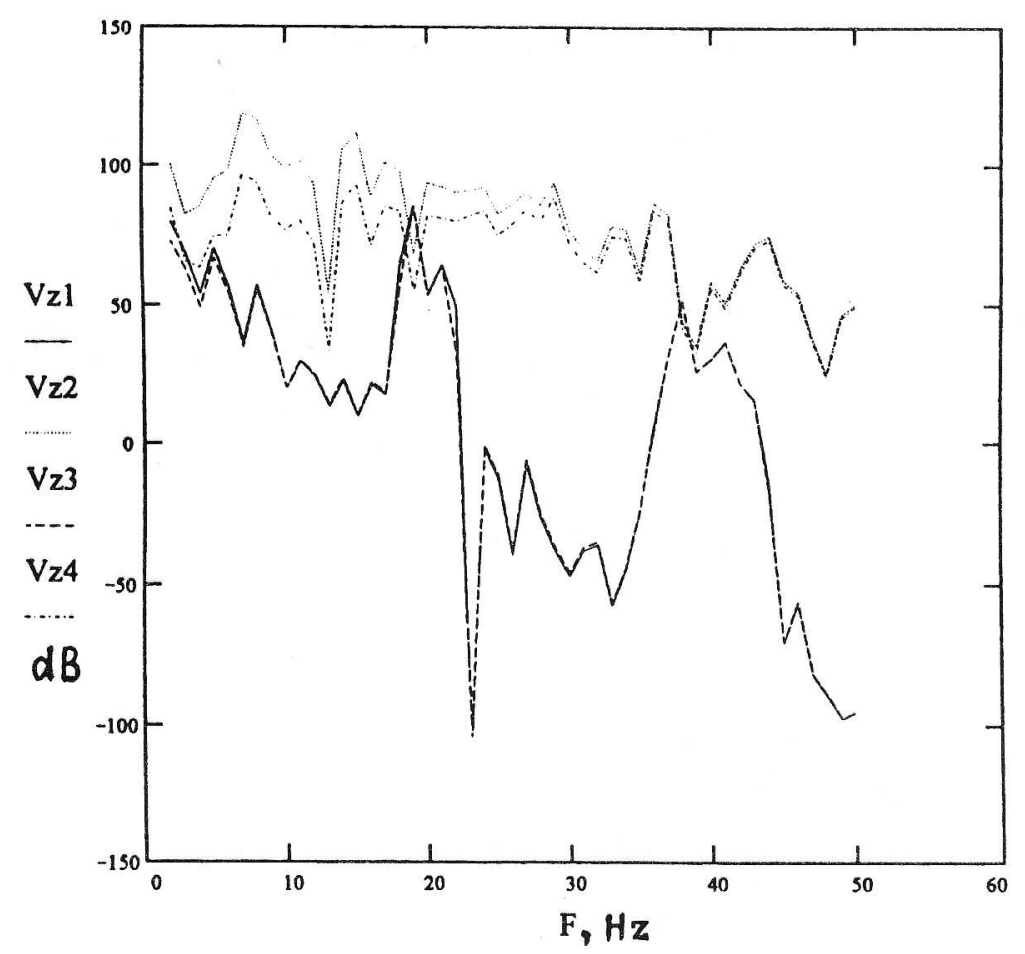

Figure 4. Ground vibration spectra (in $\mathrm{dB}$, relative to the reference level of $10^{-9} \mathrm{~m} / \mathrm{s}$ ) generated by complete TGV or Eurostar trains travelling on homogeneous ground for both sub-Rayleigh and transRayleigh train speeds (respectively: $\mathrm{v}=50 \mathrm{~km} / \mathrm{h}$ - curve $\mathrm{Vz} 1$ and $\mathrm{v}=500 \mathrm{~km} / \mathrm{h}$ - curve $\mathrm{Vz} 2$ ) and on layered ground for the same speeds (curves $\mathrm{Vz} 3$ and $\mathrm{Vz} 4$ respectively)

One can see that for homogeneous ground (curves Vz1 and Vz2) the averaged ground vibration level from a train moving at trans-Rayleigh speed $500 \mathrm{~km} / \mathrm{h}(138.8 \mathrm{~m} / \mathrm{s})$ is approximately $70 \mathrm{~dB}$ higher than from travelling at speed $50 \mathrm{~km} / \mathrm{h}(13.8 \mathrm{~m} / \mathrm{s})$. Effect of layered ground, however, results in decrease of ground vibration level from a trans-Rayleigh train at low frequencies (curve Vz4). Note, that for trains travelling at low speed effect of layered structure is small (curves $\mathrm{Vz} 1$ and $\mathrm{Vz} 3$ are almost indistinguishable).

\section{CONCLUSIONS}

Increase in speeds of railway trains is generally accompanied by increased levels of generated ground vibrations. Especially large increase in ground vibration amplitudes occurs for trans-Rayleigh trains, i.e., for trains travelling at speeds larger than Rayleigh wave velocity in the ground. Calculations performed for French TGV trains or Eurostar trains show that the average increase of about $70 \mathrm{~dB}$ takes place as compared with conventional trains.

Effect of layered geological structure of the ground results in reshaping of ground vibration spectra generated by trans-Rayleigh trains. Since Rayleigh wave velocities in layered ground are dispersive and normally increase at lower frequencies associated with deeper penetration of surface wave energy into the ground, the trans-Rayleigh condition $\mathrm{v}>\mathrm{c}_{\mathrm{R}}$ may not hold at very low frequencies. This will cause a noticeable reduction in low frequency components of generated ground vibration spectra. 


\section{REFERENCES}

Belzer A.I. (1988) "Acoustics of Solids", Springer-Verlag, Berlin, Heidelberg.

Biryukov S.V., Gulyaev Yu.V., Krylov V.V. and Plessky V.P. (1995) "Surface Acoustic Waves in Inhomogeneous Media", Springer Series on Wave Phenomena, Vol. 20, Springer-Verlag, Berlin, Heidelberg.

Brockley C.A. (1992) "The influence of track support structure and locomotive traction characteristics on short wavelength corrugations", Wear, 153, pp. 315 322.

Dawn T.M. (1983) "Ground vibrations from heavy freight trains", Journ. of Sound and Vibration, 87, pp. 351-356.

Ewing W.M., Jardetzky W.S., Press F. (1957) "Elastic waves in layered media", McGraw-Hill, New York.

Fryba L. (1973) "Vibration of solids and structures under moving loads", Noordhoff, Groningen, The Netherlands.

Graff K.F. (1975), "Wave motion in elastic solids”, Clarendon Press, Oxford.

Gutoski T.G., Dym C.L. (1976) "Propagation of ground vibration: a review", Journ. of Sound and Vibration, 49, pp. 179-193.

Jones C.J.C. (1994) "Use of numerical models to determine the effectiveness of anti-vibration systems for railways", Proc. Instn. Civ. Engrs Transp., 105, pp. $43-51$.

Jones D.V., Petyt M. (1993) "Ground vibration in the vicinity of strip load: an elastic layer on an elastic half-space", Journ. of Sound and Vibration, 161, pp. $1-18$.

Krylov V.V. (1994) "On the theory of railway-induced ground vibrations", Journ. de Physique IV, 4, C5-769-772.

Krylov V.V. (1995) "Generation of ground vibrations by superfast trains", Applied Acoustics, 44, pp. 149-164.

Krylov V.V. (1996) "Vibrational impact of high-speed trains. I. Effect of track dynamics”, Journ. Acoust. Soc. Am., 100 (5), pp. 3121-3134.

Krylov V.V., FERGUSON C.C. (1994) "Calculation of low-frequency ground vibrations from railway trains”, Applied Acoustics, 42, pp. 199-213.

Melke J. (1988) "Noise and vibration from underground railway lines: Proposals for a prediction procedure", Journ. of Sound and Vibration, 120, pp. 391-406.

Newland D.E., Hunt H.E.M. (1991) "Isolation of buildings from ground vibration: a review of recent progress", Proc. Instn. Mech. Engrs. 205, pp. 39-52. 
Remington P.J., Kurzweil L.G., Towers D.A. (1987) “Transportation noise", Reference book, Ed. by P. Nelson, Butterworths, London e.a., 1987.

Timoshenko S. (1942) "Strength of Materials", Part 2, Van Nostrand, New York.

Volberg G. (1983) "Propagation of ground vibrations near railway tracks", Journ. of Sound and Vibration, 87, pp. 371-376.

Weitsman Y. (1970) "On foundations that react in compression only", Transactions of the ASME: Journal of Applied Mechanics, 92, pp. 1019-1030.

White J.E. (1965) "Seismic Waves: Radiation, Transmission and Attenuation", McGraw-Hill, New York. 